\title{
Editorial
}

\section{Structural Damage Modelling and Assessment 2014}

\author{
Anaxagoras Elenas, ${ }^{1}$ Yuri Petryna, ${ }^{2}$ and Nawawi Chouw $^{3}$ \\ ${ }^{1}$ Department of Civil Engineering, Democritus University of Thrace, GR-67100 Xanthi, Greece \\ ${ }^{2}$ Department of Civil Engineering, Technical University Berlin, D-13355 Berlin, Germany \\ ${ }^{3}$ Department of Civil and Environmental Engineering, The University of Auckland, Auckland, New Zealand
}

Correspondence should be addressed to Anaxagoras Elenas; elenas@civil.duth.gr

Received 9 July 2015; Accepted 9 July 2015

Copyright (c) 2015 Anaxagoras Elenas et al. This is an open access article distributed under the Creative Commons Attribution License, which permits unrestricted use, distribution, and reproduction in any medium, provided the original work is properly cited.

Damage modelling and assessment is a growing field of fundamental research and a practical problem at the same time. A rapid progress in damage assessment has become possible due to new mathematical approaches developed in the last years. Combined with efficient computer simulations, they are able to predict structural damage at various scales from micromechanics to real large-scale structures. Recent developments enable efficient structural design, health monitoring, and lifetime management. New approaches to damage simulation and assessment traditionally stay also in focus of the present issue.

H. Liu et al. present a hybrid neurogenetic algorithmbased procedure for damage identification of urban overpass. Change rate of frequency and strain ratio is treated as input parameters. The optimized weight and threshold are incorporated as the initial value of ANNs to identify the structural damage condition. The proposed procedure is able to identify the location and the severity of damage (both for single-damage and for multidamage cases). The numerical technique is applied on the Qianjin Bridge. The results reveal that the proposed method can successfully identify the locations and severities of damage.

$\mathrm{X}$. Li et al. present an experimental investigation on the deformation process of a sand-filled cylindrical shell. One side of the shell experienced a contact explosive load. The shapes of the deformation were analysed. An analytical approach has been developed based on the rigid plastic hinge theory. A significant agreement has been achieved between the calculated and the experimental results. Thus, the analytical approach can be considered as a reliable tool in describing the deformation mechanism and predicting the deformation shapes of the sand-filled cylindrical shell under a lateral contact explosion.

$\mathrm{H}$. He et al. present an evolution model based on Diffusion-Limited Aggregation (DLA) for the durability analysis, considering the coupling action of the chloride ion erosion and the carbonization. The evolutionary process of the chloride ion erosion, the carbonization, and the coupling action are simulated based on the chloride ion diffusion equation and carbonation depth equation. The DLA model presented has been applied on a reinforced concrete bridge structure.

H. Zhou et al. present an improved model of the slant crack on a microbeam, based on the fracture mechanics theory. The nonlinear performance of microbeams with a bending induced slant crack of different geometry parameters is compared and investigated with regard to the dynamic behaviours. The effects of the slant crack and the electric actuation of an electrostatically actuated fixed-fixed microbeam on the dynamic characteristics are examined in detail. It is concluded that the crack position has more significant influence in the pull-in voltage value than the slant angle or the depth ratio. Approaching the slant crack to the fixed end or enlarging the external incentives will amplify the nonlinearity of the microbeam system, while the effects of depth ratio and slant angle are dependent on the crack position.

H. Lin et al. present a new method based on the deformation-energy for characterizing damage to high strength concrete structures. The material model incorporates several nonlinearities like stiffness and strength degradation under a cyclic loading. The proposed model was 
embedded into the IDARC program. An example of a high strength RC multistorey frame was used to evaluate the structural damage under various seismic excitations. The degree of structural damage was predicted satisfactorily.

By compiling these papers, the editors hope that the readers will benefit from the latest development in the field of structural damage modelling and assessment of civil engineering structures.

Anaxagoras Elenas Yuri Petryna Nawawi Chouw 


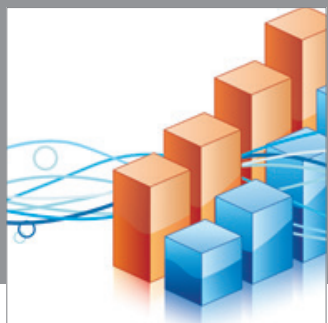

Advances in

Operations Research

mansans

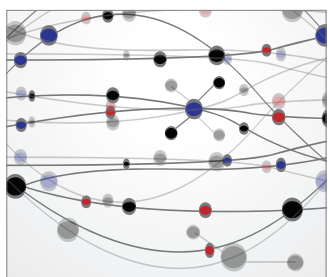

The Scientific World Journal
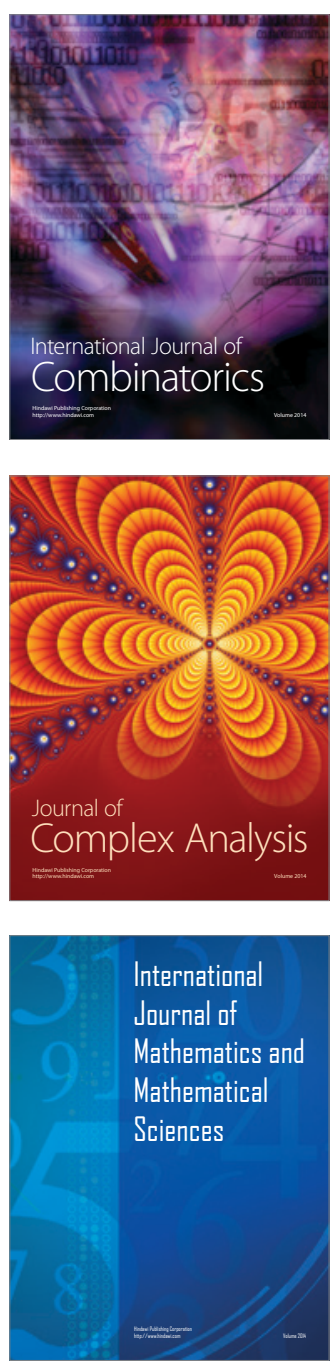
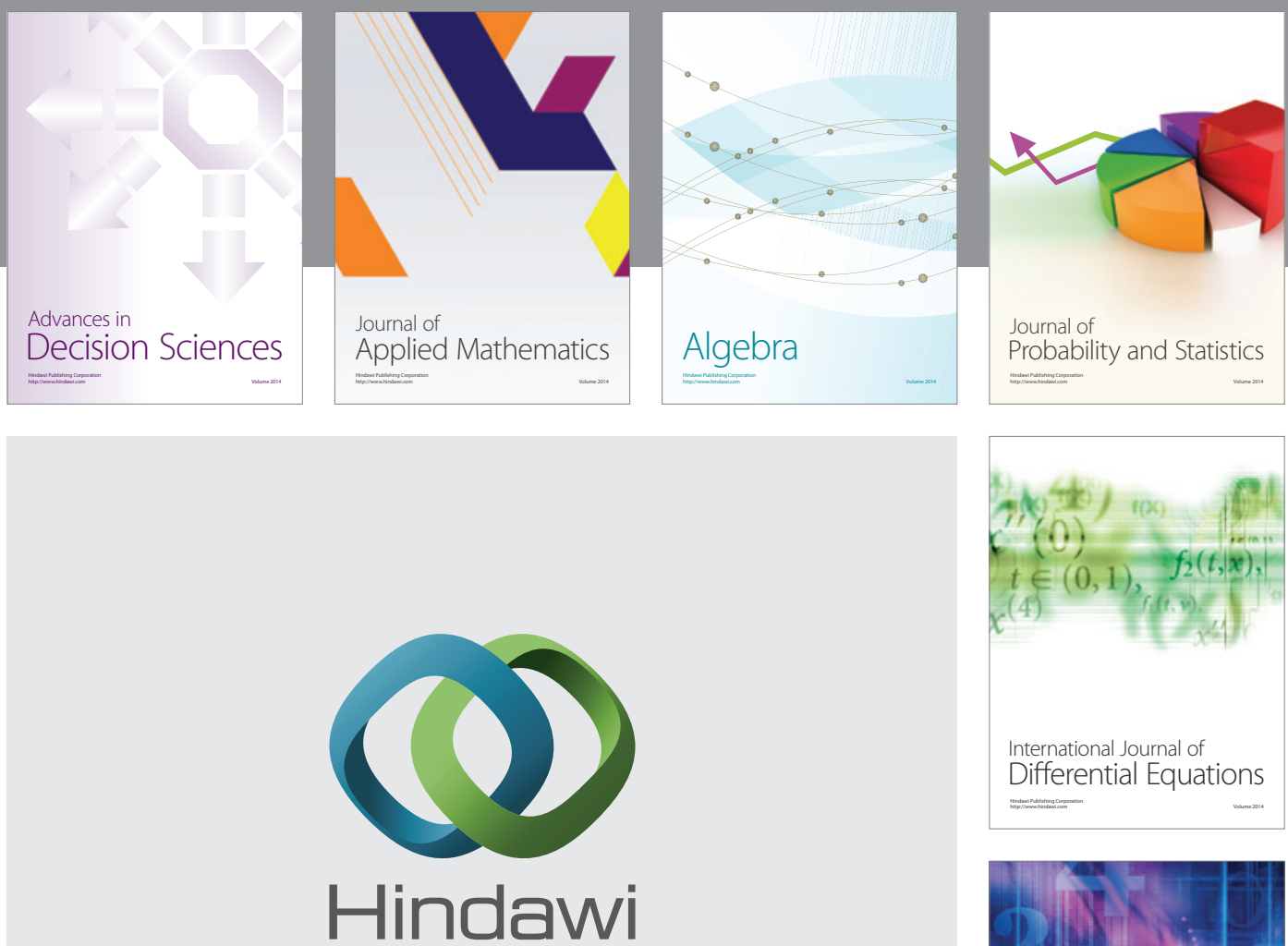

Submit your manuscripts at http://www.hindawi.com
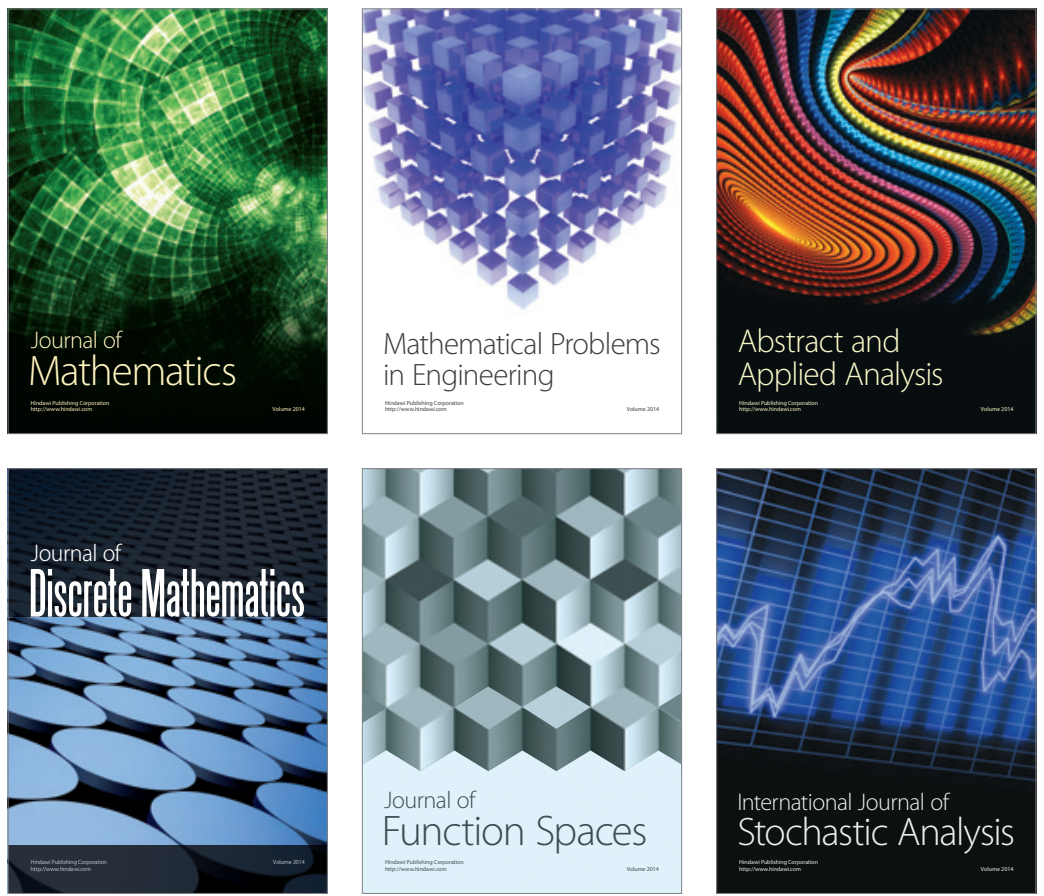

Journal of

Function Spaces

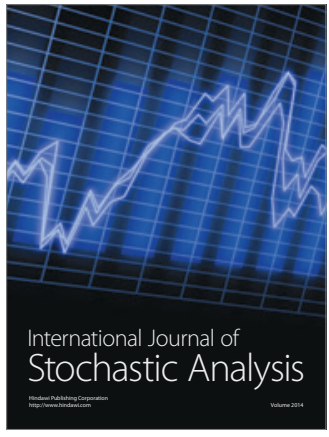

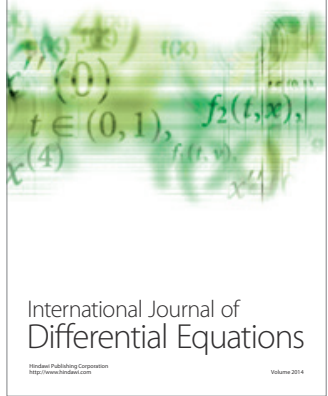
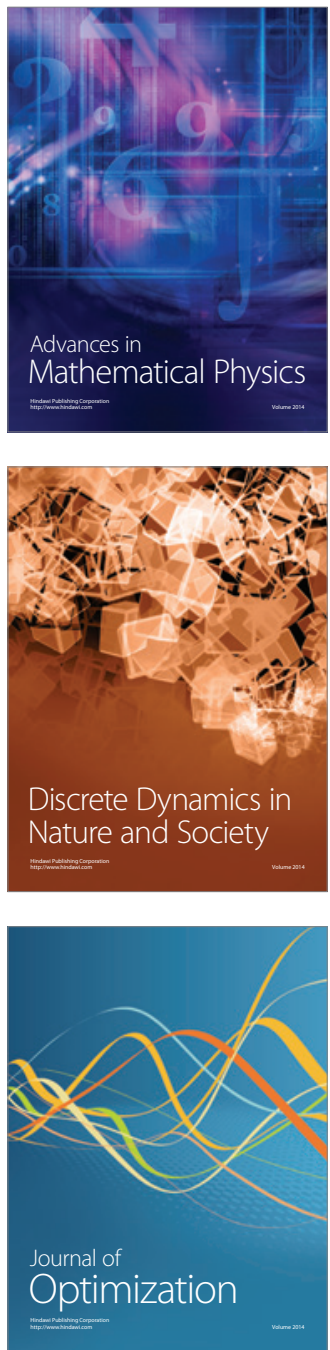